\title{
Cotton Fever: A Condition Self-Diagnosed by IV Drug Users
}

\author{
Ashley Michelle Zerr, MS IV, Kimberly Ku, MD, and Areeba Kara, MD
}

The presentation of fever in an intravenous drug user prompts diagnostic testing targeted at identifying infectious etiologies. However, an alternate diagnosis exists in "cotton fever." While few reports describe this phenomenon in the peer-reviewed literature, the diagnosis is well recognized among the intravenous drug user community. Although its etiology is not well understood, cotton fever seems to be a self-limited, febrile response to the intravenous administration of a drug filtered through cotton. Educating clinicians regarding cotton fever may limit unnecessary hospital admissions and improve our ability to care for this population. (J Am Board Fam Med 2016;29:276-279.)

Keywords: Cotton Fever, Intravenous Drug Abuse

A 26-year-old woman with a 2-year history of intravenous (IV) drug use presented to the emergency department (ED) after the sudden onset of chills, fever, abdominal pain, chest pain, and headache immediately after injecting heroin that was prepared through cotton filtration. She denied previous similar episodes and stated that this was the first time she had used cotton for filtration. She reported injecting heroin 20 times daily, using about $1 \mathrm{~g}$ /day. She also used tobacco, but denied the use of alcohol or other illicit drugs. Upon arrival she attributed her current symptoms to "cotton fever," which she had heard mentioned among heroin users. She denied any medical or family history, and took no prescribed medications.

\section{Physical Examination}

At presentation she appeared to be in acute distress. She was febrile, with a temperature of $38.6^{\circ} \mathrm{C}$, and tachycardic, with a heart rate of $137 \mathrm{bpm}$. Blood

This article was externally peer reviewed.

Submitted 24 August 2015; revised 1 December 2015; accepted 7 December 2015.

From the Internal Medicine Residency (KK) and the Department of Medicine (AK), Indiana University School of Medicine (AMZ), Indianapolis; and Inpatient Medicine, IU Health Methodist Hospital, Indianapolis, IN (AK).

Funding: none.

Conflict of interest: none declared.

Corresponding author: Areeba Kara, MD, Inpatient Medicine, Noyes Pavilion Suite E120, Indianapolis, IN 46202 (E-mail: akara@iuhealth.org). pressure was $129 / 47 \mathrm{mmHg}$, respiratory rate 19 breaths/minute, and oxygen saturation 99\% breathing ambient air. The remainder of the physical examination demonstrated no abnormalities with the exception of mild bilateral, lowerquadrant abdominal tenderness on palpation, flushed skin, and multiple injection marks along both arms. Specifically, no murmurs, Janeway lesions, Osler's nodes, Roth spots, or splinter hemorrhages were observed.

\section{Diagnostic Workup}

Initial laboratory results, including a complete blood count and basic metabolic profile, demonstrated the following: white blood cell count, $2000 \mathrm{k} / \mathrm{mm}^{3}$; alkaline phosphatase, $145 \mathrm{U} / \mathrm{L}$; total bilirubin, $1.1 \mathrm{mg} / \mathrm{dL}$; and direct bilirubin, 0.3 $\mathrm{mg} / \mathrm{dL}$. A urine drug screen was positive for barbiturates and opiates. Hepatitis $\mathrm{C}$ antibody testing was positive; human immunodeficiency virus (HIV) screening antibody was negative. Electrocardiography showed sinus tachycardia, and chest radiography was unrevealing. A stringlike echo-density without an attachment point was seen in the right atrium on a transthoracic echo, suggesting a valvular vegetation or thrombus. Bilateral upper- and lower-extremity ultrasounds were performed for further evaluation and revealed an acute deep venous thrombosis of the right brachial vein. 


\section{Initial Diagnosis and Treatment}

The patient's presentation was consistent with systemic inflammatory response syndrome (heart rate, $>90 \mathrm{bpm}$; temperature, $>38^{\circ} \mathrm{C}$; white blood cell count, $\left.<4,000 \mathrm{k} / \mathrm{mm}^{3}\right) .{ }^{1}$ Given her history of IV drug use, blood and urine cultures were obtained and broad-spectrum antibiotics (vancomycin and piperacillin-tazobactam) were initiated. Based on the results of the Doppler studies showing a deep venous thrombosis, anticoagulation with heparin was initiated. She refused transesophageal echocardiography but was amenable to focused, repeat transthoracic echocardiography. This study concluded that the initial echodensity was a prominent chiari network, a normal variant.

\section{Outcome}

Within 4 hours of admission, the patient was afebrile and had no recurrence of fever. The following day she was agitated and complained of lacrimation, generalized pain, and diarrhea, which was attributed to opioid withdrawal. Symptomatic treatment with benzodiazepines and clonidine was initiated.

She grew increasingly agitated and signed out against medical advice on the second day of hospitalization. Final cultures were negative. The diagnosis of cotton fever was established given her transient fever and the exclusion of infective endocarditis and other pathology to explain her presentation with systemic inflammatory response syndrome.

\section{Discussion}

Many IV drugs, including heroin, are heated before being drawn into a syringe for injection. Once heated, users can draw the drug through a cotton ball into a syringe as an inexpensive method of filtering the drug. Users may also attempt to extract the drug from previously used cotton when their heroin supply is low. This was termed "shooting the cottons" by Shragg" in 1978. Cotton fever refers to the transient elevation in temperature within minutes after injection of drug filtered through cotton, or after shooting the cottons. Symptoms include fever, chills, headache, abdominal pain, nausea, vomiting, and muscle aches. ${ }^{2}$

Three theories have been proposed to explain the pathophysiology of cotton fever. The immunologic theory proposes that individuals have preformed antibodies against the cotton that cause a transient reaction. The pharmacologic theory proposes that pyrogenic substances within the cotton cause a febrile response when introduced into the bloodstream. The current leading theory is the endotoxin theory, which proposes that the Gram-negative bacteria Enterobacter agglomerans colonizes the cotton plant and releases an endotoxin that causes transient fever. ${ }^{3}$ Support for this theory comes from a positive blood culture of E. agglomerans in 1 patient who brought the same cotton to the hospital for culture, which grew E. agglomerans, Leclercia adecarboxylata, and Acinetobacter lwoffi. ${ }^{4}$

Table 1. Comparison of Reported Cotton Fever Cases

\begin{tabular}{|c|c|c|c|c|c|c|c|c|c|c|}
\hline Reference & $\begin{array}{c}\text { Age } \\
\text { (Years) }\end{array}$ & Sex & IV Drug & $\begin{array}{l}\text { Time Until } \\
\text { Symptom } \\
\text { Onset (min) }\end{array}$ & $\begin{array}{l}\text { Temperature } \\
\text { upon Arrival } \\
\left({ }^{\circ} \mathrm{C}\right)\end{array}$ & $\begin{array}{c}\mathrm{HR} \\
(\mathrm{bpm})\end{array}$ & $\begin{array}{c}\mathrm{BP} \\
(\mathrm{mmHg})\end{array}$ & $\begin{array}{c}\text { WBC } \\
\text { Count at } \\
\text { Admittance } \\
\left(\mathrm{k} / \mathrm{mm}^{3}\right)\end{array}$ & $\begin{array}{l}\text { Time Until } \\
\text { Symptom } \\
\text { Resolution } \\
\text { (hours) }\end{array}$ & Disposition \\
\hline 2 & 23 & $\mathrm{~F}$ & Heroin & 10 & 38.5 & 130 & NA & 21,700 & 12 & AMA \\
\hline 2 & 22 & M & Heroin & 15 & 40.3 & 132 & NA & 5,800 & 7 & DC \\
\hline 3 & 33 & $\mathrm{~F}$ & $\begin{array}{l}\text { Pentazocine and } \\
\text { methylphenidate }\end{array}$ & $\begin{array}{l}\text { A few } \\
\text { minutes }\end{array}$ & 36 & 110 & $140 / 90$ & 4,200 & 12 & DC \\
\hline 4 & 38 & M & Heroin & 10 & 39.1 & 96 & $99 / 38$ & 2,800 & NA & $\mathrm{DC}$ \\
\hline 5 & 36 & M & Dilaudid & 120 & 38 & 122 & $120 / 58$ & 11,600 & 48 & DC \\
\hline 6 & 22 & M & Heroin & 30 & 39.4 & 120 & $123 / 80$ & 9700 & 12 & DC \\
\hline 7 & 22 & $\mathrm{~F}$ & Heroin & 20 & 39 & 101 & $106 / 64$ & 22,600 & 24 & DC \\
\hline $\begin{array}{l}\text { This } \\
\text { article }\end{array}$ & 26 & $\mathrm{~F}$ & Heroin & $\begin{array}{l}\text { A few } \\
\text { minutes }\end{array}$ & 38.6 & 137 & $129 / 47$ & 2,000 & 5 & AMA \\
\hline
\end{tabular}

AMA, left against medical advice; BP, blood pressure; DC, discharged; F, female; HR, heart rate; IV, intravenous; M, male; NA, not available; WBC, white blood cell. 
Many similarities exist among the reported cases of cotton fever, including presentation, time to symptom onset, and resolution (Table 1). Symptoms often manifest within 20 minutes of drug injection: fever, chills, headache, abdominal pain, and muscle aches. At presentation to the ED, a patient will usually be febrile, often in mild acute distress, and meet systemic inflammatory response syndrome criteria. Laboratory results may demonstrate leukocytosis but are otherwise nonspecific. Electrocardiography and chest radiography will demonstrate no abnormalities with the exception of sinus tachycardia. Blood cultures and transthoracic echocardiography are commonly performed to rule out infective endocarditis and are negative in cotton fever. Symptoms usually resolve within 12 hours of onset. ${ }^{2-7}$

Despite the benign course of cotton fever, it is important to rule out sources of sepsis, infective endocarditis, and other conditions associated with IV drug use, including HIV and hepatitis. Depending on the patient's presentation, IV antibiotics may be initiated until data from blood cultures is available. Management of drug intoxication or withdrawal is important during the observation period. Counseling, including chemical dependency consultation and patient education, should also be offered. Other than supportive care, no specific intervention is required for cotton fever. Positive results of additional testing such as HIV require further follow-up. Cotton fever may reoccur if the patient continues to use cotton filtration with injections.

The transient nature of the condition has led to the proposal that when cotton fever is suspected, patients can have blood cultures drawn and be monitored in the ED. If the diagnosis is cotton fever, symptoms should resolve or improve within 12 hours, and the patient could be discharged with follow-up. This approach may decrease the rate of hospitalizations and thus have potential cost-saving benefits. ${ }^{3}$ However, $25 \%$ of patients (2 of 8 ) in the reported cases left against medical advice, raising concern that adequate follow-up may not feasible within this population.

Our patient's accurate recognition of her diagnosis is not the first time this has been documented in the literature. In another case report, upon discharge after a negative workup, a patient conjectured that she had cotton fever. ${ }^{7}$ The first mention of cotton fever that we could locate in peer-re- viewed publications was from $1975 .{ }^{8}$ There have been fewer than a dozen total publications on this condition since then (Table 1). By contrast, although we cannot ascertain the exact date cotton fever was recognized by the IV drug user community, information on the diagnosis is widely available on online forums, for example, "Drugs Forum," "Heroin Helper," and "Bluelight." Many users on these sites report having cotton fever multiple times and suggest symptomatic relief with aspirin, benzodiazepines, sleep, herbal teas, and acetaminophen. Users also recommend seeking medical attention if symptoms do not resolve within 24 hours. $^{9-11}$

\section{Conclusion}

The discrepancy of knowledge between patients and providers regarding the recognition and management of cotton fever is increasingly important as the IV drug use epidemic continues. Familiarity with the diagnosis will allow practitioners to expand their differential diagnosis when encountering a febrile illness in an IV drug user, encourage more detailed history taking regarding drug use habits, and potentially allow observation rather than hospitalization for some patients. Of note, this case occurred in Indiana around the same time as the HIV outbreak in southern Indiana related to needle sharing during the injection of oxymorphone. ${ }^{12}$ The state of Indiana has enacted a needleexchange program in an attempt to decrease the transmission of both HIV and hepatitis, as the rates of both have surged in the past year. ${ }^{13}$ In a similar fashion, with an increasing recognition of cotton fever, there may be a role for interventions targeted at both providers and IV drug users to manage and prevent cotton fever.

\section{References}

1. Levy MM, Fink MP, Marshall JC, et al; International Sepsis Definitions Conference. 2001 SCCM/ ESICM/ACCP/ATS/SIS International Sepsis Definitions Conference. Intensive Care Med 2003;29: 530-8.

2. Shragg T. "Cotton fever" in narcotic addicts. JACEP 1978;7:279-80.

3. Harrison DW, Walls RM. "Cotton fever": a benign febrile syndrome in intravenous drug abusers. J Emerg Med 1990;8:135-9.

4. Ferguson R, Feeney C, Chirugi VA. Enterobacter agglomerans-associated cotton fever. Arch Intern Med 1993;153:2381-2. 
5. Ramik D, Mishriki YY. The other "cotton fever." Infect Dis Clin Pract 2008;16:192-3.

6. Torka P, Gill S. Cotton fever: an evanescent process mimicking sepsis in an intravenous drug abuser. J Emerg Med 2013;44:385-7.

7. Xie Y, Pope BA, Hunter AJ. Cotton fever: does the patient know best? J Gen Intern Med 2015 June 24. [Epub ahead of print]

8. Thomson BD. Medical complications following intravenous heroin. Ariz Med 1975;32:798-801.

9. Cotton fever/dirty hit - ever had it? How to avoid [discussion forum on the Internet]. Drugs-Forum.com. Available from: https://drugs-forum.com/forum/ showthread.php? $\mathrm{t}=69686$ \&highlight $=$ Cotton + fever . Accessed January 15, 2016.

10. Dr. H. Cotton fever. Heroin Helper [c2001, modified January 10, 2004]. Available from http://www. heroinhelper.com/user/health/cotton_fever.shtml. Accessed January 15, 2016.

11. DeLee. What is cotton fever? [comment posted April 25, 2011, in discussion forum on the Internet]. Bluelight.org. Available from: http://www.bluelight. org/vb/threads/428437-What-is-cotton-fever. Accessed January 15, 2016.

12. Conrad C, Bradley HM, Broz D, et al; Centers for Disease Control and Prevention (CDC). Community outbreak of HIV infection linked to injection drug use of oxymorphone-Indiana, 2015. MMWR Morb Mortal Wkly Rep 2015;64:443-4.

13. Indiana State Department of Health. Spotlight semi-annual report - December 2014. Spotlight on HIV/STD/viral hepatitis. Indiana semi-annual report. January 2015. Available from: http://www.in. gov/isdh/26638.htm. Accessed January 15, 2016. 